\title{
Importância do monitoramento e disponibilização de dados hidrológicos para a gestão integrada dos recursos hídricos
}

\author{
Importance of monitoring and availability of hydrological data for \\ integrated water resources management
}

\section{Marco Alésio Figueiredo Pereira ${ }^{1}$ Bruno Lippo Barbieiro ${ }^{2}$ Daniela Muller de Quevedo ${ }^{3}$}

\section{Palavras-chave:}

Aquisição de dados hidrológicos Estações hidrométricas

Comitês de bacias hidrográficas

\begin{abstract}
Resumo
A conservação do meio ambiente, com a implementação de práticas visando o desenvolvimento sustentável e abordando a gestão integrada dos recursos naturais, é essencial para a manutenção do meio e da sociedade. Nesse sentido, este trabalho teve por objetivo avaliar a possibilidade de implementar a Gestão Integrada de Recursos Hídricos - GIRH, a partir de dados hidrológicos disponibilizados no endereço eletrônico do Sistema Nacional de Informações sobre Recursos Hídricos (SNIRH) sob a responsabilidade da Agência Nacional de Águas - ANA. Para isso, o estudo apresenta as condições do monitoramento hidrométrico da bacia hidrográfica do Rio dos Sinos - RS e discutindo a importância da disponibilização de dados hidrológicos para a GIRH. A partir de informações disponibilizadas no SNIRH, fez-se uma pesquisa sobre as séries históricas de estações pluviométricas, fluviométricas e de qualidade da água instaladas na respectiva bacia, sendo avaliado se, a partir do monitoramento hidrométrico realizado, é possível a implementação da GIRH. Foi possível identificar que, a partir dos dados existentes, a implementação e a manutenção de uma GIRH fica comprometida. Constata-se que existem diversas entidades que monitoram os recursos hídricos na bacia, no entanto, quando disponibilizados, os dados observados por tais entidades são armazenados em sites específicos de cada instituição ou nem mesmo são disponibilizados, o que acarreta dificuldade de aquisição de dados para os usuários finais. Além disso, verifica-se a necessidade da ampliação da rede de monitoramento e a implantação de uma rede automática e telemétrica de monitoramento na bacia.
\end{abstract}

\footnotetext{
1 Universidade Feevale - Programa de Pós-graduação em Qualidade Ambiental. Novo Hamburgo - RS, Brasil marco@feevale.br

2 Universidade Feevale - Bolsista de Iniciação Científica. Novo Hamburgo - RS, Brasil. brunolipp7@gmail.com

3 Universidade Feevale - Programa de Pós-graduação em Qualidade Ambiental. Novo Hamburgo - RS, Brasil. danielamq@feevale.br
} 


\section{Keywords:}

Hydrological data acquisition

Hydrometric stations

River basin committees

\begin{abstract}
The environment conservation with sustainable development implantation practices to integrated natural resource management is essential for the maintenance of the environment and society. Thus, this article aims to evaluate the hydrometric monitoring of Sinos River basin, in Rio Grande do Sul state, and argue the availability importance of hydrological data for integrated water resources management - IWRM, based on hydrological data disponibility at the National Water Resources Information System - SNIRH, under the responsibility of the National Water Agency ANA. For this, the study presents the conditions of the hydrometric monitoring Sinos River watershed and discussing the importance of making hydrological data available to IWRM. Based on information made available in the SNIRH, a survey was carried out on the historical series of rainfall, flow and water quality in stations installed in the respective basin, being evaluated whether from the hydrometric monitoring carried out, it is possible to implement IWRM. It was possible to identify that from the existing data the IWRM implementation and maintenance are compromised. Several entities monitor water resources in the basin, however, when made available, the data observed by such entities are stored on specific sites of each institution or are not even made available, which makes it difficult to acquire data for end-users. Besides, there is a need to expand the monitoring network and implement an automatic and telemetric monitoring network in the basin.
\end{abstract}

\section{INTRODUÇÃO}

A ausência de planejamento para a utilização dos recursos naturais, em especial dos recursos hídricos $(\mathrm{RH})$, muitas vezes por causa da ilusão da farta disponibilidade, faz com que o uso de técnicas inadequadas acabe gerando um ciclo de consequências negativas, resultando em perdas econômicas, sociais e ambientais, consequentemente, ocasionando o declínio do desenvolvimento e do bem-estar social. Schewe et al. (2014) apontam que, devido ao crescimento populacional e econômico esperado para as próximas décadas, a demanda por $\mathrm{RH}$ tende a crescer e a intensificar os problemas relacionados a esses recursos, tanto em regiões que já sofrem quanto em regiões que passarão a sofrer com eventos de escassez. Uma forma de tentar contornar, adequadamente, essa possível situação é gerir os RH e o meio ambiente como um todo, através da Gestão Integrada dos Recursos Hídricos (GIRH) (SHRUBSOLE et al., 2017; VEALE; COOKE, 2017; LEIDEL et al., 2012; WMO, 2009; BISWAS, 2004).

A GWP (2000) define que a GIRH deve promover o desenvolvimento e a gestão da água, da terra e dos recursos conexos, a fim de maximizar o bem-estar econômico e social de forma equitativa sem comprometer a sustentabilidade de ecossistemas vitais. Benham et al. (2011) abordam que a gestão de bacias hidrográficas pode ser caracterizada por um contínuo, geograficamente definido, integrado, processos colaborativos de criação e implementação de planos, programas e projetos desenvolvidos para sustentar e melhorar as bacias hidrográficas e seus ecossistemas de funcionamento.

Nesse sentido, para o êxito de uma adequado plano de gestão, o monitoramento contínuo e preciso (espacial e temporal) e a avaliação de seus dados são fundamentais em todas as fases. WMO (2009) ressalva que, sem o monitoramento sistemático, seguido pela avaliação, a oportunidade de aprender com a experiência é reduzida assim como a oportunidade de fazer ajustes à luz de novas informações, de conhecimentos e da experiência. De acordo com a GWP (2000), a avaliação das condições dos $\mathrm{RH}$ deve abordar a ocorrência das águas superficiais e subterrâneas em uma dinâmica espaço-temporal, tendo por objetivo dar uma avaliação preliminar dos requisitos da água para o desenvolvimento assumido.

Desta forma, o objetivo deste artigo foi avaliar se é possível implementar a GIRH, a partir de dados hidrológicos disponibilizados no endereço eletrônico do Sistema Nacional de Informações sobre Recursos Hídricos (SNIRH) sob a responsabilidade da Agência Nacional de Águas - ANA e, propor locais estratégicos para 
instalação de estações de monitoramento. Como estudo de caso, utilizou-se estações de monitoramento pluviométrico, fluviométrico e de qualidade da água locadas na bacia hidrográfica do Rio dos Sinos - RS.

\section{GESTÃO DOS RECURSOS HÍDRICOS}

\section{Comitês de bacias hidrográficas (CBH)}

A primeira experiência de um país desenvolvido a concentrar esforços para o problema da poluição hídrica ocorreu no início do século XX, na bacia do rio Ruhr - Alemanha, quando foi criada a Associação de Água da Bacia do Ruhr, em 1913. Naquele momento, foi estabelecido, em assembleia, que todos os seus associados (empresas, indústrias, comércio, municípios e comunidade) iriam seguir a política de uso da água, inclusive, pagando pelo uso e pela poluição das águas (RIBEIRO, 2006). Outra experiência interessante foi a francesa, cuja política de gestão implementada tem servido de modelo para diversos países do mundo, inclusive para o Brasil. Esse modelo é um dos percursores na aplicação da cobrança pelo uso da água combinada com uma gestão participativa e integrada da bacia hidrográfica. Lanna (1997) comenta que, entre as diversas legislações introduzidas para a gestão dos $\mathrm{RH}$ franceses, a principal foi a Lei $1245 / 64$, que definiu a bacia hidrográfica como unidade básica de gerenciamento. Para a implementação da gestão participativa, foram definidos órgãos colegiados, que tinham o poder de gestão e aprovação de programas. Esses órgãos eram denominados de "Comitês de Bacia Hidrográfica".

De acordo com Porto e Porto (2008), no Brasil, os primeiros registros de comitês de bacias hidrográficas foram feitos a partir da complexa e eminente demanda das condições sanitárias das bacias do Alto Tietê e Cubatão, no estado de São Paulo. Em 1978, foi constituído o Comitê Especial de Estudos Integrados de Bacias Hidrográficas (CEEIBH) e, posteriormente, foram criados os comitês executivos das bacias do Paraíba do Sul, do São Francisco e do Ribeira de Iguape. A partir de então, surgiram diversas iniciativas de gestão descentralizada, como o Consórcio Intermunicipal Santa Maria/Jacu, no estado do Espírito Santo, e os consórcios de Piracicaba, Capivari e Jundiaí, no estado de São Paulo. Em 1988, a partir da iniciativa da comunidade, com o apoio do Estado, surgiram os Comitês de Bacias Sinos e Gravataí, afluentes do rio Guaíba, no estado do Rio Grande do Sul, sendo esta considerada a primeira experiência brasileira de instituição de comitê de bacia tendo como referência o modelo francês (ANA, 2011). A Figura 1 mostra a evolução da implementação de comitês de bacias hidrográficas estaduais e interestaduais no contexto nacional.

Correlacionando variáveis geográficas, demográficas, socioeconômicas e de saneamento versus estados brasileiros que possuem $\mathrm{CBHs}$, Feil et al. (2017) concluíram que a existência de comitês de bacias hidrográficas relaciona-se com o tamanho da população, que promove maior pressão à medida que aumenta. A existência de comitês de bacias hidrográficas também se deve ao crescimento econômico, à quantidade de conflitos decorrentes dos múltiplos usos, da coleta de esgotos e do nível de degradação da qualidade ambiental.

Santos et al. (2015); Dourojeanni (2002) e Lanna (1996) salientam que o comitê de bacia é um ente do Estado, com caráter normativo, deliberativo e consultivo, que tem poder de tomada de decisão em situações em que coexistem muitos interesses individuais $\mathrm{e}$ coletivos. Tendo os comitês de bacias as prerrogativas elencadas, eles devem agir como órgão representativo dos diversos usuários da água, tanto públicos quanto privados, agindo com lisura em suas decisões. Conforme Senra e Nascimento (2017), dentro da GIRH, deve-se considerar uma gama mais ampla das partes interessadas dos setores relacionados à água, não apenas os tradicionais "profissionais da água”, o que favorece uma visão mais holística.

De acordo com a PNRH, o CBH é um órgão essencial para a implementação, para a manutenção e para o desenvolvimento de uma política articulada entre os diversos níveis de gestão no âmbito municipal, estadual e nacional. Assim sendo, o CBH possui como escopo a gestão dos recursos hídricos de uma unidade territorial denominada bacia hidrográfica. A bacia hidrográfica, de acordo com a PNRH, é a unidade básica para o planejamento e a gestão dos $\mathrm{RH}$. Watanabe et al. (2014) afirmam que a tomada de decisão sobre ações a serem implementadas para a gestão dos RH na bacia hidrográfica tem de ser bem avaliada, pois, uma vez processadas as mudanças, seu retorno ao estado anterior é bem mais difícil, devido às perdas e aos ganhos decorrentes dos processos (ambientais e sociais) subsequentes. Os autores supracitados declaram também que o processo de tomada de decisão precisa ser capaz de incluir componentes para a política, para as organizações e para as dimensões sociais e questões financeiras. Korfmacher (2001) adverte que os principais fatores que ameaçam a credibilidade da gestão 
junto aos diferentes usuários são grupos em desvantagem representativa em relação a outros, a não representatividade, a disputa de poder, entre outros. Ao encontro disso, Rauber e Cruz (2013) abordam que, para a gestão da água, deve-se utilizar a gestão integrada de bacia hidrográfica, com foco nos usos múltiplos e no compartilhamento da responsabilidade sobre sua quantidade e qualidade.

Figura 1 - Evolução da criação de comitês de bacias hidrográficas no Brasil no período de 1988 a 2010.

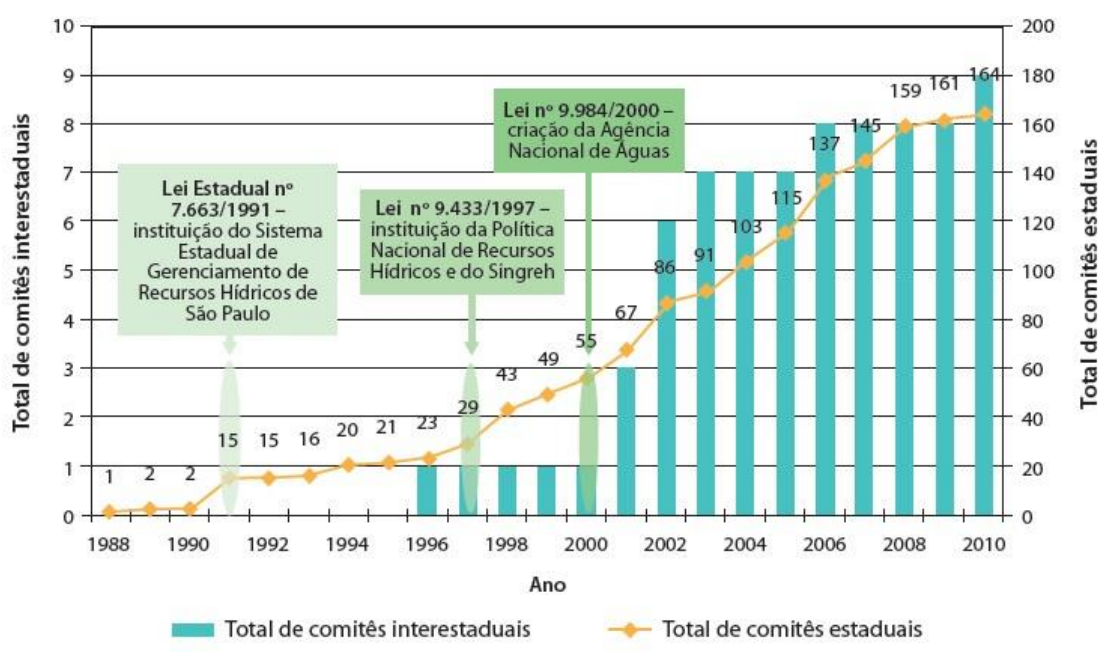

Fonte: Adaptado de ANA (2011).

\section{Importância do monitoramento hidrométrico}

Uma premissa para o sucesso da implementação e da manutenção da GIRH é o conhecimento da quantidade e da qualidade da água disponível na bacia em estudo. Assim sendo, o monitoramento hidrológico é de suma importância para a determinação do balanço hídrico e do conhecimento das inter-relações e das agregações dos diversos processos envolvidos; desde a entrada de água na bacia em suas diversas formas, precipitação, neve e granizo, passando pela evaporação e pela evapotranspiração, pela infiltração, pela percolação, pelo armazenamento da água no solo, pela formação do escoamento subsuperficial e superficial, pela geração da vazão, pelos processos de erosão, pelo transporte e deposição dos sedimentos, pelo transporte de substâncias orgânicas e inorgânicas, até seu deflúvio na foz da bacia.

A WMO (2008) afirma que o objetivo final da coleta de dados hidrológicos, sejam eles medições de precipitação, registros de nível de água, medições de descarga, monitoramento de águas subterrâneas ou amostragem de qualidade de água, é fornecer um conjunto de dados (indicadores) com qualidade suficiente para que possam ser usados na tomada de decisão em todos os aspectos da gestão dos $\mathrm{RH}$, na vasta gama de aplicações operacionais bem como na investigação.

O monitoramento em campo configura-se como uma tarefa árdua e dispendiosa, pois a implementação de equipamentos de medições e o treinamento de equipes de campo aptas a realizar o monitoramento hidrométrico requer investimento. Apesar das dificuldades apresentadas, Stahli et al. (2011) defendem que uma longa série histórica de precipitação e de vazão é essencial para determinar conclusões acertadas sobre o comportamento e a tendência que uma bacia hidrográfica pode sofrer em função das mudanças climáticas e de uso do solo. Assim, comprova-se a importância do monitoramento constante com a implementação de equipamentos que realizem a mensuração do dado de forma contínua e em um intervalo de tempo que consiga mensurar com fidedignidade os fenômenos hidrometeorológicos envolvidos.

Sendo esse conjunto de dados de grande valor intrínseco, o monitoramento dos dados hidrológicos é, portanto, um trabalho importante em si, devendo ser realizado de forma eficaz, a fim de maximizar os resultados dos investimentos colocados para sua aquisição e sua possibilidade de ser efetivamente utilizado. A gestão dos dados pode ser abordada a partir de dois pontos de vista complementares: um visando à preservação da perda e da degradação, 
tornando-o acessível e disseminando os conjuntos de dados que foram coletados, e outro visando aumentar seu valor para os usuários finais, o que inclui consistência dos dados e preenchimento de falhas, para, assim, gerar dados calculados (por exemplo, evaporação ou descarga líquida) ou agregados (por exemplo, médias de descargas médias) (WMO, 2008). A Figura 2 apresenta um organograma dos procedimentos a serem adotados para a implementação, operação e disponibilização de dados hidrológicos.

Figura 2 - Organograma de implantação, operação, armazenamento, transmissão e disponibilização de dados de uma rede de monitoramento hidrológico.

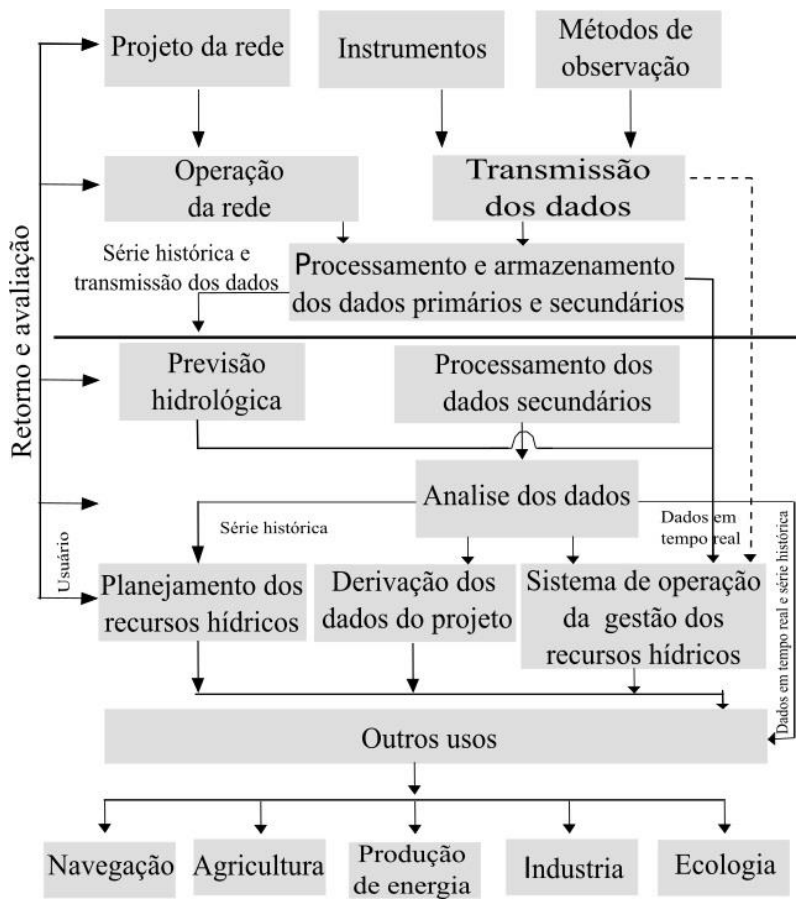

Fonte: Adaptado de WMO (2009).

\section{Equipamentos automáticos de medição}

A partir da década de 80 com o expansão da informática e da telecomunicação as informações hidrológicas puderam ser adquiridas em tempo real, ocasionando um significativo avanço na medição dos dados; isso aconteceu devido ao processo de automatização dos equipamentos de medições. Dentre as variáveis monitoradas a precipitação e o nível do rio puderam ser medidos de forma mais discretizada, possibilitando, consequentemente, o conhecimento dos fenômemos hidrológicos de forma mais acurada.

Atualmente o pluviógrafo automático mais utilizado é o de cubas basculantes, neste equipamento a água recolhida por um cilindro, com área de captação de $400 \mathrm{~cm}^{2}$, é dirigida para um conjunto de duas cubas articuladas por um eixo central, fazendo um efeito gangorra conforme se enche e se esvazia cada cuba, o aparelho registra o número de movimentos e o tempo em que ocorre cada movimento. Cada basculhada apresenta um volume de $0,25 \mathrm{~mm}$. O diferencial deste equipamento é possuir um data logger capaz de armazenar uma série de dados por um tempo finito. Essa capacidade de armazenamento se dará conforme o tempo de aquisição dos dados, ou seja, quanto menor for o intervalo de tempo entre um registro e outro, menor será a capacidade de armazenamento do logger. Geralmente o intervalo de medição se dá conforme o grau de detalhamento necessário, em virtude do tempo de concentração da bacia e do tempo de visita da equipe técnica ao equipamento. $\mathrm{O}$ conjunto é composto pelo pluviógrafo, propriamente dito, por um data logger, por uma bateria de alimentação e um painel solar. Informações detalhadas sobre equipamentos automáticos e formas indiretas de medição como radares meteorológicos e sensoriamento remoto podem ser encontradas em WMO (2017). Os equipamentos automáticos apresentam, entre outras vantagens, a possibilidade de mensurar com maior fidedignidade a intensidade, duração e horário da precipitação.

Para medição dos níveis dos rios, há diversos princípios de medição desde sensores de 
boia, sensores ultrassônicos e sensores de pressão da coluna d'água, sendo este último o mais utilizado. Este tipo de sensor mede a coluna d'água sobre ele. As oscilações do nível são registradas, em tempos pré-determinados, com excessão do sensor de nível os demais componentes são os mesmos do sistema pluviométrico. Informações pormenores sobre os diversos tipos de sensores podem ser encontrada em WMO (2010). Tanto os dados de precipitação quanto de nível do rio podem ser transmitidos remotamente, quando possuem equipamentos transmissores específicos (modem). A transmissão remota dos dados, estação telemétrica, é essencial para um planejamento preventivo para uma bacia hidrográfica, pois a partir destes pode ser implementado um sistema de alerta para prevenção de desastres naturais, como deslizamento, enxurradas e inundações (REIS et al. 2016). No Brasil, de acordo com o Sistema de Alerta de Eventos Críticos - SACE, 16 bacias hidrográficas possuem um sistema de alerta hidrológico em operação. Além desta potencialidade, o monitoramento automatizado proporciona outros benefícios, melhorando a qualidade dos dados, através de uma coleta com intervalos menores e no mesmo horário em todas as estações da rede; proporcionando, consequentemente, uma visão holística das condições hidrológicas da bacia hidrográfica.
Dados acurados quando utilizados como fontes alimentadoras para modelos hidrológicos, potencializam a representatividade do modelo.

\section{ESTUDO DE CASO, BACIA DO RIO DOS SINOS}

A bacia hidrográfica do Rio dos Sinos situa-se a nordeste do estado do Rio Grande do Sul, possui área aproximada de $3.746,68 \mathrm{~km}^{2}$, abrange 32 municípios e apresenta uma população estimada em 1.350.000 habitantes (Figura 3). Os principais rios que drenam a bacia são o Rio Rolante, o Rio da Ilha, o Rio Paranhana e o Rio dos Sinos. Este último tem sua nascente no município de Caraá e sua foz no delta do Jacuí. Em virtude de seu grau de desenvolvimento socioeconômico, a bacia apresenta diversos problemas relacionados aos $\mathrm{RH}$ e ao meio ambiente, porque a demanda pelo uso da água para abastecimento, uso industrial e irrigação, muitas vezes é maior do que a disponibilidade. Seus afluentes e seu rio principal recebem grandes quantidades de resíduos industriais e domésticos não tratados, além de serem contaminados por agrotóxicos (PETRY et al., 2016; BENVENUTI et al., 2015).

Figura 3 - Localização da bacia do Rio dos Sinos e espacialização das estações pluviométricas, fluviométricas e de qualidade da água na bacia.

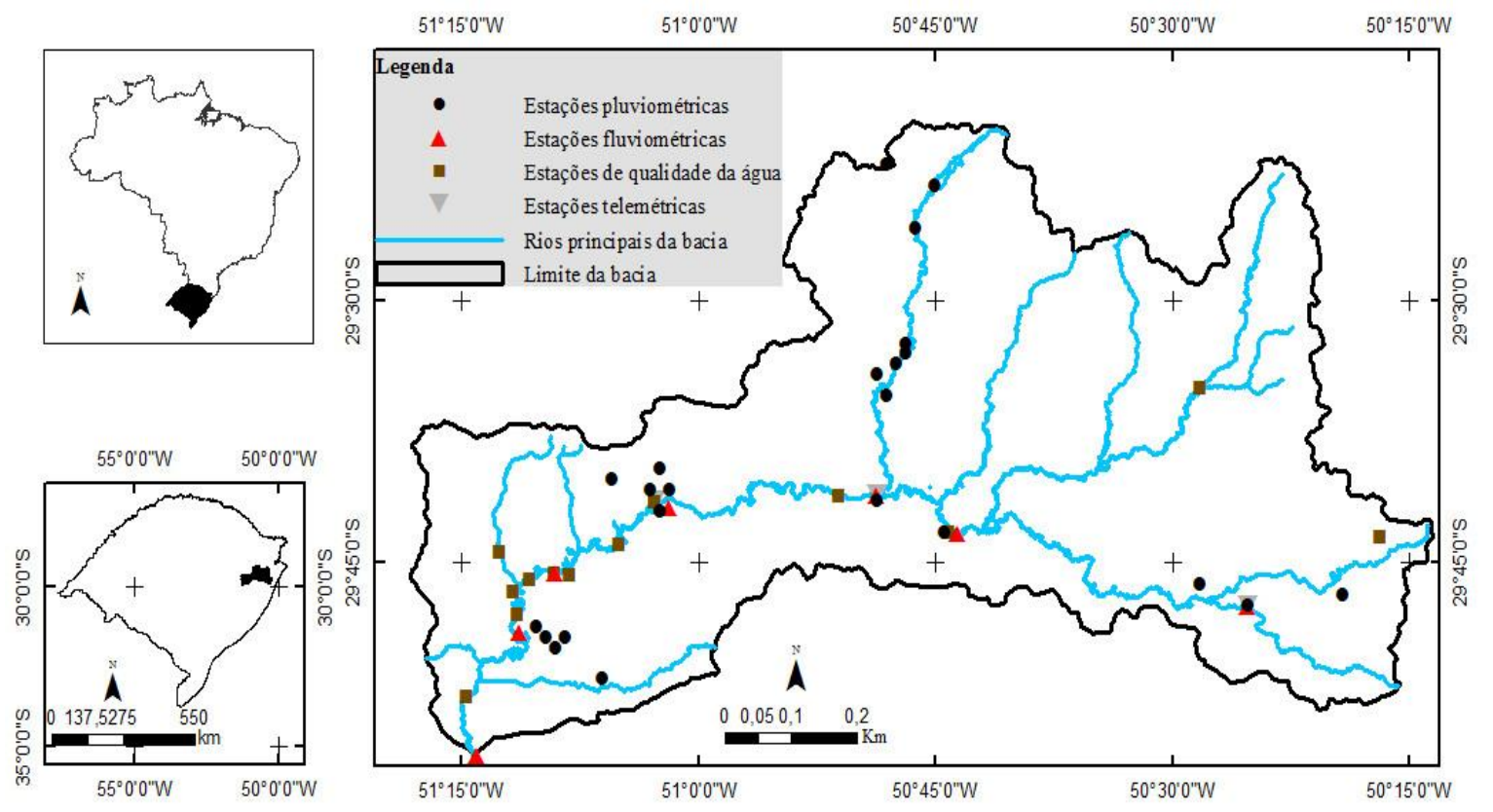

Fonte: org. dos autores (2017). 
Os dados foram obtidos do site da ANA (Agência Nacional de Águas) no Sistema de Informações Hidrológicas (HidroWeb). Esse endereço eletrônico é uma ferramenta integrante do Sistema Nacional de Informações sobre Recursos Hídricos (SNIRH), em que é disponibilizado o acesso ao banco de dados referente às informações coletadas pela Rede Hidrometeorológica Nacional (RHN). Para a verificação do conjunto de dados disponíveis (precipitação, cota, vazão e qualidade da água), fez-se uma pesquisa verificando as estações pluviométricas, fluviométricas e de qualidade da água locadas na respectiva bacia, Figura 3. Salienta-se que foram consideradas somente as estações que apresentavam sua condição como "em operação" para a análise da GIRH. O período de acesso ao respectivo site foi de agosto de 2017 a março de 2018.

\section{Monitoramento Pluviométrico}

Dados de precipitação, coletados de forma contínua e sem falhas, são essenciais para a determinação do comportamento espaçotemporal das chuvas. Kaiser e Porto (2005) comentam que tradicionalmente a chuva é medida em postos pluviométricos, mas também pode ser medida de forma indireta por Sensoriamento Remoto, através de satélites e radares meteorológicos.

Os dados referentes ao monitoramento pluviométrico na bacia do Rio dos Sinos, disponibilizados no site supracitado, são coletados de forma direta, com pluviômetros convencionais e automáticos. Das 23 estações exibidas na Figura 3, somente quatro apresentam dados disponíveis para download, conforme Tabela 1. Sendo que as estações de Canela e de Campo Bom estão desativas. Assim, somente as estações Taquara Montante e Sapucaia do Sul possuem dados disponíveis e em operação.

Tabela 1 - Dados básicos das estações pluviométricas.

\begin{tabular}{|c|c|c|c|c|c|c|}
\hline Código & Nome & Município & $\begin{array}{c}\text { Entidade } \\
\text { responsável }\end{array}$ & $\begin{array}{c}\text { Latitude } \\
(\mathrm{S})\end{array}$ & $\begin{array}{c}\text { Longitude } \\
\text { (O) }\end{array}$ & $\begin{array}{c}\text { Série } \\
\text { histórica }\end{array}$ \\
\hline 2950009 & Canela & Canela & CEEE & $29^{\circ} 48^{\prime} 00^{\prime \prime}$ & $50^{\circ} 48^{\prime} 00^{\prime \prime}$ & $\begin{array}{c}01 / 05 / 41- \\
31 / 12 / 78\end{array}$ \\
\hline 2950068 & Taquara Montante & Taquara & ANA & $29^{\circ} 43^{\prime} 15^{\prime \prime}$ & $50^{\circ} 44^{\prime} 06^{\prime \prime}$ & $\begin{array}{c}01 / 10 / 09- \\
01 / 01 / 18\end{array}$ \\
\hline 2951028 & Sapucaia do Sul & Sapucaia do Sul & ANA & $29^{\circ} 49^{\prime} 12^{\prime \prime}$ & $51^{\circ} 09^{\prime} 40^{\prime \prime}$ & $\begin{array}{c}01 / 01 / 64- \\
01 / 01 / 18\end{array}$ \\
\hline 2951069 & Campo Bom & Campo Bom & INMET & $29^{\circ} 41^{\prime} 00^{\prime \prime}$ & $51^{\circ} 02^{\prime} 00^{\prime \prime}$ & $\begin{array}{c}01 / 01 / 85 \\
31 / 12 / 98\end{array}$ \\
\hline
\end{tabular}

Fonte: org. dos autores (2018).

\section{Monitoramento fluviométrico}

O monitoramento fluviométrico de uma estação contempla a medição do nível do rio - cota (m), a medição de descarga líquida - $Q\left(\mathrm{~m}^{3} / \mathrm{s}\right)$, a medição de descarga sólida - Qs (ton/dia), o levantamento do perfil transversal da seção de medição $(m)$ e a medição dos parâmetros de qualidade da água $Q A$. Esses parâmetros são correlacionados entre si, no entanto, nem sempre todos são medidos. O acompanhamento da variação do nível do rio no decorrer do tempo pode ser realizado de forma convencional com réguas linimétricas ou de forma automática através de sensores (linígrafos). A vazão pode ser determinada de forma direta com medições realizadas em campo, indiretamente através da equação da curvachave, ou então, através de séries temporais sintéticas (DETZEL et al., 2013) e por imagens de satélites - Sensoriamento Remoto. Sichangi et al. (2016) e Birkinshaw et al. (2014) relatam que o Sensoriamento Remoto para a determinação de fluxos, principalmente em grandes bacias, tem sido utilizado com sucesso por diversos pesquisadores. De acordo com a WMO (2010), registros de vazão são os dados básicos usados como fontes confiáveis para o conhecimento da variabilidade espaço temporal do fluxo. Os dados obtidos a partir do monitoramento contínuo são extremamente úteis, pois esses registros poderão servir como auxílio para a elaboração de estudos científicos e de projetos de engenharia, definindo usos prioritários e ações para a conservação do meio ambiente. Os dados de cota e vazão disponibilizados em http://www.snirh.gov.br/hidroweb, referentes às estações locadas na bacia do Rio dos Sinos, são oriundos de medições diretas e indiretas obtidas pela equação da curva-chave. Na Tabela 2, visualiza-se as estações que compõem o 
monitoramento na bacia. Das estações elencadas, constata-se que somente as estações de Campo Bom (83377800), de São Leopoldo (87382000) e de Taquara Montante (87374000) monitoram todos os parâmetros apresentados. Somente três estações emitem seus dados via telemetria e três estações apresentam dados disponibilizados para download no endereço eletrônico citado.

Tabela 2 - Estações fluviométricas em operação na bacia do Rio dos Sinos.

\begin{tabular}{|c|c|c|c|c|c|c|c|}
\hline $\begin{array}{l}\text { Código da } \\
\text { estação }\end{array}$ & 87318000 & 87380000 & 87376000 & 87393000 & 87382000 & 87385000 & 87374000 \\
\hline $\begin{array}{l}\text { Nome da } \\
\text { estação }\end{array}$ & $\begin{array}{l}\text { Arroio } \\
\text { Caraá }\end{array}$ & $\begin{array}{l}\text { Campo } \\
\text { Bom }\end{array}$ & $\begin{array}{l}\text { Foz do } \\
\text { Paranhana }\end{array}$ & $\begin{array}{l}\text { Foz do rio } \\
\text { dos Sinos } \\
59\end{array}$ & $\begin{array}{l}\text { São } \\
\text { Leopoldo }\end{array}$ & $\begin{array}{l}\text { Siderurgica } \\
\text { Riograndense }\end{array}$ & $\begin{array}{l}\text { Taquara } \\
\text { Montante }\end{array}$ \\
\hline Responsável & SEMA-RS & ANA & SEMA-RS & DMAE & ANA & DEPRC & ANA \\
\hline Município & Caraá & $\begin{array}{l}\text { Campo } \\
\text { Bom }\end{array}$ & Taquara & Canoas & $\begin{array}{l}\text { São } \\
\text { Leopoldo }\end{array}$ & $\begin{array}{l}\text { Sapucaia do } \\
\text { Sul }\end{array}$ & Taquara \\
\hline $\begin{array}{l}\text { Última } \\
\text { atualização }\end{array}$ & 08/04/2016 & 05/07/2017 & 08/04/2016 & $25 / 04 / 2006$ & 05/07/2017 & $25 / 04 / 2006$ & 05/07/2017 \\
\hline Ativas & Sim & Sim & Sim & Sim & Sim & Sim & Sim \\
\hline $\begin{array}{l}\text { Cotas } \\
\text { médias }\end{array}$ & Sim & Sim & Sim & Sim & Sim & Sim & Sim \\
\hline $\begin{array}{l}\text { Vazão } \\
\text { média }\end{array}$ & Sim & Sim & Não & Não & Sim & Não & Sim \\
\hline Sedimentos & Não & Sim & Não & Não & Sim & Não & Sim \\
\hline $\begin{array}{l}\text { Qualidade } \\
\text { da água }\end{array}$ & Não & Sim & Não & Não & Sim & Não & Sim \\
\hline Telemétrica & Sim & Sim & Sim & Não & Não & Não & Não \\
\hline $\begin{array}{l}\text { Latitude } \\
\text { (S) }\end{array}$ & $29^{\circ} 47^{\prime} 24^{\prime \prime}$ & $29^{\circ} 41^{\prime} 24^{\prime \prime}$ & $29^{\circ} 41^{\prime} 24^{\prime \prime}$ & $29^{\circ} 55^{\prime} 48^{\prime \prime}$ & $29^{\circ} 45^{\prime} 36^{\prime \prime}$ & $29^{\circ} 49^{\prime} 12^{\prime \prime}$ & $29^{\circ} 43^{\prime} 12^{\prime \prime}$ \\
\hline $\begin{array}{l}\text { Longitude } \\
\text { (O) }\end{array}$ & $50^{\circ} 25^{\prime} 12^{\prime \prime}$ & $51^{\circ} 03^{\prime} 00^{\prime \prime}$ & $50^{\circ} 48^{\prime} 36^{\prime \prime}$ & $51^{\circ} 13^{\prime} 48^{\prime \prime}$ & $51^{\circ} 09^{\prime} 00^{\prime \prime}$ & $51^{\circ} 10^{\prime} 48^{\prime \prime}$ & $50^{\circ} 44^{\prime} 24^{\prime \prime}$ \\
\hline $\begin{array}{l}\text { Série } \\
\text { histórica }\end{array}$ & $\begin{array}{l}\text { Sem dados } \\
\text { para } \\
\text { download }\end{array}$ & $\begin{array}{c}01 / 11 / 1939 \\
- \\
01 / 01 / 2018\end{array}$ & $\begin{array}{l}\text { Sem dados } \\
\text { para } \\
\text { download }\end{array}$ & $\begin{array}{l}\text { Sem dados } \\
\text { para } \\
\text { download }\end{array}$ & $\begin{array}{c}01 / 07 / 1973 \\
- \\
01 / 10 / 2017\end{array}$ & $\begin{array}{l}\text { Sem dados } \\
\text { para } \\
\text { download }\end{array}$ & $\begin{array}{c}01 / 08 / 1996 \\
- \\
01 / 01 / 2018 \\
\end{array}$ \\
\hline
\end{tabular}

Fonte: org. dos autores (2018).

\section{Monitoramento de qualidade da água}

Com o avanço da sociedade, a demanda por $\mathrm{RH}$ aumentou consideravelmente, em consequência, o monitoramento teve de acompanhar essa evolução. Além da parte quantitativa, a disponibilidade de $\mathrm{RH}$ também está intrinsecamente ligada à qualidade da água, já que a poluição das fontes de água pode inviabilizar diferentes tipos de usos (WWAP, 2017). Nesse sentido, o monitoramento da qualidade da água tem como principal objetivo verificar se as condições físico-químicas da água se adequam ao uso pretendido. WMO (2013) apresenta uma série de ações que o monitoramento proporciona diretamente na qualidade da água, extremamente úteis para definir as potencialidades de uso e para tomar decisões referentes ao GIRH. Em relação ao monitoramento da qualidade da água na bacia do Rio dos Sinos, a Tabela 3 apresenta estações que realizam tal monitoramento bem como os parâmetros monitorados e o número de medições de cada parâmetro. Das estações apresentadas, nota-se que as estações de Novo Hamburgo (87380015) e de São Leopoldo (87382000) apresentam um número maior de parâmetros e de dados medidos, no entanto, eles ainda apresentam uma pequena série histórica. Salienta-se que a estação de São Leopoldo (87382000) apresenta discrepância entre o número de medições de cada parâmetro, ficando evidenciado que o monitoramento de qualidade da água não é contínuo. Na estação de Novo Hamburgo (87380015), não é realizada medição de descarga líquida, consequentemente, impossibilitando a determinação da carga poluente, o que é uma falha para a determinação da efetiva contaminação do meio.

Já as estações de Campo Bom (87380000) e de Taquara Montante (87374000) monitoram apenas os parâmetros de temperatura da água e da amostra, $\mathrm{pH}$, turbidez, condutividade elétrica e oxigênio dissolvido, parâmetros medidos, 
geralmente, quando ocorre a medição de descarga líquida na estação.

As estações Campo Bom (87377800), Caraá (87318500), Parobé (87376800), Portão (87382020), São Leopoldo (87380030), São
Leopoldo (87381800), São Leopoldo (87382010) e Sapucaia do Sul (87382025) por apresentarem somente duas medições, de 2016 a 2017, não foram apresentadas na Tabela 3 .

Tabela 3 - Parâmetros de qualidade da água medidos na bacia do Rio dos Sinos.

\begin{tabular}{|c|c|c|c|c|}
\hline Código da estação & 87380000 & 87380015 & 87382000 & 87374000 \\
\hline Nome da estação & Campo Bom & Novo Hamburgo & São Leopoldo & $\begin{array}{l}\text { Taquara } \\
\text { Montante }\end{array}$ \\
\hline Responsável & ANA & FEPAM & ANA & ANA \\
\hline Série Histórica & $\begin{array}{c}11 / 02 / 2000- \\
23 / 01 / 2018\end{array}$ & $\begin{array}{c}01 / 01 / 2001 \text { - } \\
17 / 04 / 2017\end{array}$ & $\begin{array}{c}12 / 07 / 1976- \\
24 / 01 / 2018\end{array}$ & $\begin{array}{c}09 / 02 / 2002- \\
16 / 11 / 2017\end{array}$ \\
\hline Temperatura do ar & 54 & - & 59 & 45 \\
\hline Temperatura da amostra & 54 & 36 & 88 & 47 \\
\hline $\mathrm{pH}$ & 55 & 36 & 84 & 47 \\
\hline Turbidez & 30 & 36 & 47 & 34 \\
\hline Condutividade Eletrica & 50 & - & 81 & 45 \\
\hline DQO & - & - & 15 & - \\
\hline DBO & - & 36 & 10 & - \\
\hline $\mathrm{OD}$ & 49 & 36 & 71 & 44 \\
\hline Sólidos Totais & - & 36 & 1 & - \\
\hline Sólidos Suspensos Totais & - & - & 15 & - \\
\hline Sólidos Dissolvidos Totais & - & - & 1 & - \\
\hline Cloretos & - & - & 1 & - \\
\hline Fosfato Total & - & 36 & 3 & - \\
\hline NitrogênioTotal & - & 36 & 1 & - \\
\hline Nitrogênio Amoniacal & - & 36 & 14 & - \\
\hline Nitratos & - & 36 & 14 & - \\
\hline Nitritos & - & 36 & 15 & - \\
\hline Coliformes Fecais & - & 36 & 15 & - \\
\hline Alcalinidade Total & - & - & 14 & - \\
\hline Ortofosfato Total & - & - & 14 & - \\
\hline Nitrogênio Orgânico & - & 36 & & - \\
\hline Descarga Líquida & 73 & - & 42 & 46 \\
\hline Descarga Sólida & 73 & - & 1 & 35 \\
\hline Fósforo Total & - & 36 & - & - \\
\hline Nitrogênio Total & - & 36 & - & - \\
\hline
\end{tabular}

Fonte: org. dos autores (2017)

\section{Avaliação da situação atual na bacia do Rio dos Sinos}

Existem 23 estações pluviométricas, sete estações fluviométricas e 12 estações de qualidade da água locadas ao longo da bacia (Figura 3). No entanto, conforme apresentado nas Tabelas 1, 2 e 3, constata-se que, em efetiva operação e com disponibilidade de dados, há somente duas estações pluviométricas, três estações fluviométricas e quatro estações de qualidade da água (Figura 4). Observa-se que as estações de monitoramento fluviométrico e pluviométrico estão localizadas na parte média e baixa da bacia, não havendo nenhuma estação com dados disponíveis nas partes mais altas ou em afluentes do Rio dos Sinos. Essa concentração nos locais mais a jusante ocasiona uma lacuna espaço-temporal no conhecimento qualiquantitativo dos $\mathrm{RH}$, dificultando, por exemplo, estudos de balanço hídrico bem como a localização geográfica dos pontos de contaminação orgânica e inorgânica.

Em relação às estações pluviométricas, das 23 estações apresentadas na Figura 3, somente as estações Sapucaia do Sul (02951028) e Taquara Montante (02950068) estão em operação e com dados para download (Tabela 1). Essas estações, contudo, estão localizadas na parte média e baixa da bacia. Dessa forma, não estão sendo disponibilizados os dados de precipitação que ocorrem nas cabeceiras da bacia. 
Em relação às estações fluviométricas, das sete estações monitoradas, somente as estações de Campo Bom (87380000), São Leopoldo (87382000) e Taquara Montante (87374000) apresentam seus dados de cota e de vazão disponibilizados, com uma série histórica (diária) de 78 anos, 44 anos e 22 anos, respectivamente.

Em relação às estações de qualidade da água, das 12 estações que constam no site supracitado, somente quatro estações apresentam um monitoramento relativamente contínuo, o que é considerado pouco em se tratando de uma bacia com tantos problemas ambientais.

Constata-se que, em sua maioria, os dados monitorados (precipitação, cota, vazão e qualidade da água) não apresentam uma série histórica robusta, para que seja possível tirar conclusões fidedignas do comportamento do estado trófico da bacia no decorrer do tempo e do espaço.

Devido ao rio Paranhana e o rio Rolante serem os principais afluente do Rio dos Sinos e em virtude destas sub-bacias apresentarem cidades de pequeno e médio porte no entorno dos seus rios. Sugere-se a instalação de postos fluviométricos com medição de níveis, vazão, qualidade da água e telemetria na foz dos respectivos rios, conforme Figura 4 . Em relação ao postos pluviométricos conforme demonstrado na Figura 3 existe o monitoramento das chuvas no trecho médio e superior da bacia, o que ocorre é a não disponibilização dos dados coletados no site supracitado. Já para o monitoramento da qualidade da água, recomenda-se a reativação dos pontos de monitoramento, principalmente na parte média e alta da bacia, bem como a inserção de parâmetros que diagnostifiquem o estado trófico do curso d'água. Uma forma viável e econômica para a operação da rede e disponibilização dos dados é a comunicação entre as diversas entidades operadoras do sistema. Além disso, a implementação e manutenção de uma rede hidrológica telemétrica, na bacia do Rio dos Sinos, é primordial para um monitoramento mais consistente, proporcionando informações robustas para uma adequada gestão integrada dos recursos hídricos.

Figura 4 - Dispersão das estações de monitoramento que efetivamente estão em operação e com seus dados disponibilizados para download no endereço eletrônico http://www.snirh.gov.br/hidroweb.

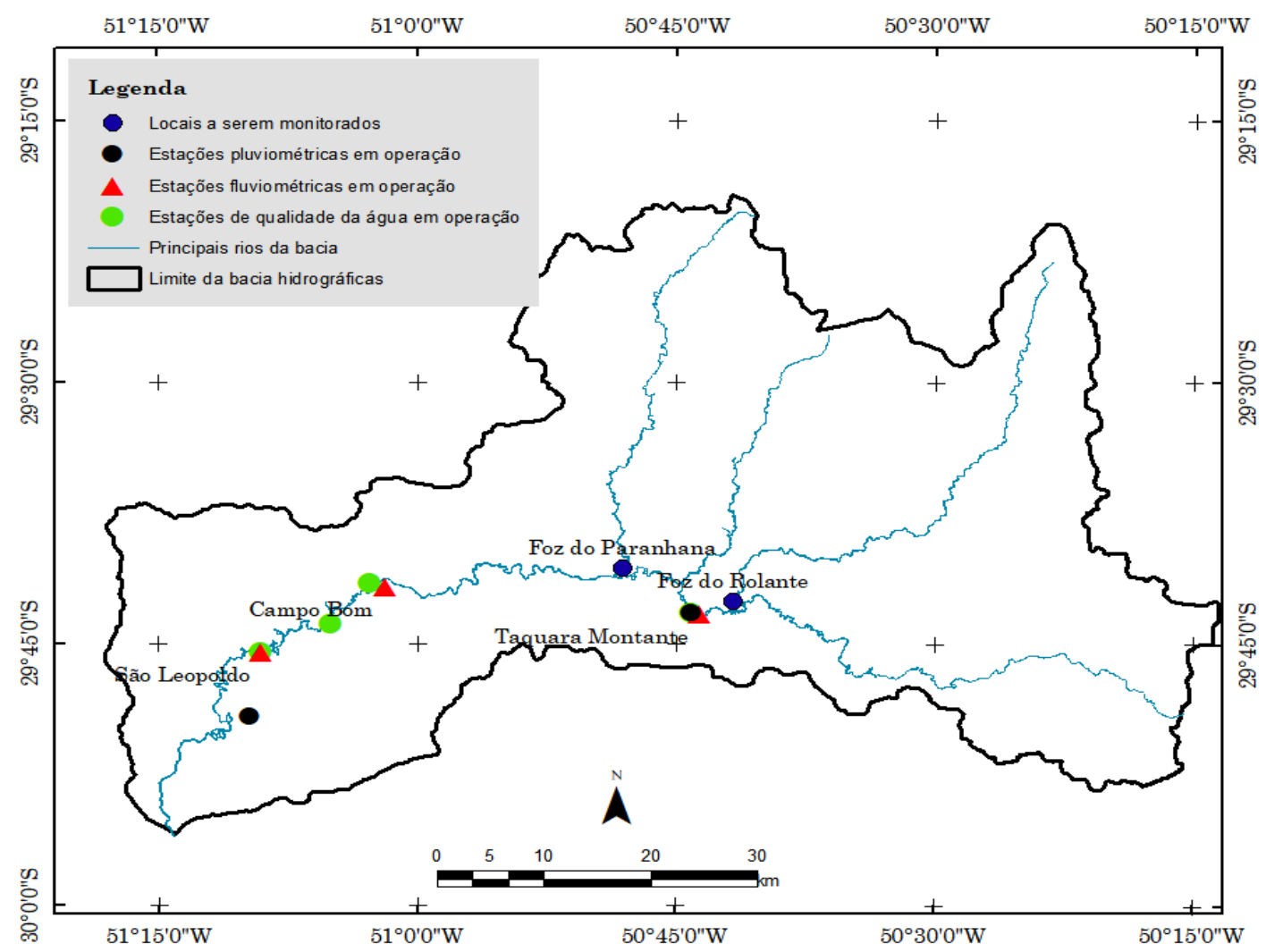

Fonte: org. dos autores (2017). 


\section{CONSIDERAÇÕES FINAIS}

A coleta e o registro de dados hidrológicos são caros, em termos de esforços e recursos (WMO, 2014), mas a sua disponibilização é de grande valia para a sociedade. Assim sendo, a continuidade do monitoramento, a implantação de novas estações e a coleta de um número adequado de parâmetros proporcionará um melhor entendimento da dinâmica espaçotemporal dos $\mathrm{RH}$ na bacia hidrográfica. Consequentemente, oferecerá subsídios para a melhor forma de implementação de uma GIRH. Além disso, o monitoramento contínuo propicia o entendimento das possíveis mudanças de longo prazo, como usos consuntivos da água, mudanças climáticas ou uso e ocupação do solo, que venham a ocorrer na bacia hidrográfica, causando a não estacionariedade de séries hidrológicas. Senra e Nascimento (2017) colocam que o não conhecimento dessas mudanças afeta o planejamento e a operação da infraestrutura hídrica para o atendimento de usos múltiplos, como geração de energia, navegação, irrigação, abastecimento de água, controle de inundação entre outros.A determinação e a coleta de dados são de grande valia para o conhecimento das condições quantitativas e qualitativas dos diversos cursos d'água que compõem a bacia do Rio dos Sinos. A disponibilização desses dados após seu devido tratamento (consistência) trarão grandes benefícios à comunidade, oportunizando a elaboração de estudos sobre as condições tróficas da bacia e proporcionando a elaboração de planos de ações para a conservação do meio ambiente e o desenvolvimento sustentável. Assim sendo, para a efetiva implantação de uma GIRH na bacia hidrográfica do Rio dos Sinos, a partir da ótica do monitoramento hidrométrico, a manutenção das estações em operação, com a disponibilização de seus dados, bem como a implementação de novas estações são questões indispensáveis para o êxito de tal empreitada. Nesse sentido, a disponibilização de recursos financeiros por parte de fontes financiadoras de amparo e de apoio à pesquisa são essenciais assim como a efetividade do PNRH.

\section{REFERÊNCIAS}

ALVES, D. D.; RIEGEL, R. P.; QUEVEDO, D. M. de, OSÓRIO, D. M. M.; COSTA, G. M. da; NASCIMENTO, C. A. do, TELÖKEN, F.; Seasonal assessment and apportionment of surface water pollution using multivariate statistical methods: Sinos River, southern Brazil. Environmental Monitoring and Assessment. v. 190, n. 384, p. 13, 2018. http://dx.doi.org/10.1007/s10661-018-6759-3

ANA - Agência Nacional de Águas (Brasil). O Comitê de Bacia Hidrográfica: o que é e o que faz? - Cadernos de capacitação em Recursos Hídricos, v.1, Brasília: 64 p. 2011. Disponível

em:http://arquivos.ana.gov.br/institucional/sge/ CEDOC/Catalogo/2012/CadernosDeCapacitaca o1.pdf>. Acesso em: 04/10/2017

BENHAM, B. L.; YAGOW, G.; CHAUBEY, I.; DOUGLAS-MANKIN, K. R.; Advances in Watershed Management: Modeling, Monitoring, and Assessment. American Society of Agricultural and Biological Engineers, v. 54, n. 6, p. 2167-2170, 2011. http://dx.doi: 10.13031/2013.40915

BENVENUTI, T.; KIELING-RUBIO, M. A.; KLAUCK, C. R.; RODRIGUES, M. A. S.; Evaluation of water quality at the source of streams of the Sinos River Basin, southern Brazil. Brazilian Journal of Biology, v. 15, n. $2, \quad$ p. $98-104,2015$. http://dx.doi.org/10.1590/1519-6984.1513

BIRKINSHAW, S. J.; MOORE, P.; KILSBY, C. G.; O'DONNELL, G. M.; HARDY, A. J.; BERRY, P. A. M.; Daily discharge estimation at ungauged river sites using remote sensing. Hydrological Processes, v.28, p. 1043-1054, 2014. http://dx.doi.org/10.1002/hyp.9647

BISWAS, A. K.; Integrated Water Resources Management: A Reassessment - A Water Forum Contribution. International Water Resources Association. Water International, v. $29, \quad$ n. $2, \quad$ p. 248-256, 2004. https://doi.org/10.1080/02508060408691775

DETZEL, D. H. M.; BESSA, M. R.; MINE, M. R. M.; Amostragem de Séries Sintéticas Hidrológicas. Revista Brasileira de Recursos Hídricos. v. 18, n. 4, p. 173-184, 2013. http://dx.doi:10.21168/rbrh.v18n4.p173184

DOUROJEANNI, A.; JOURAVLEV, A.; CHÁVEZ, G. Gestión del agua a nível de cuencas: teoría y práctica. Santiago de Chile: CEPAL, Serie recursos naturales e infraestructura, v. 47, 83 p., 2002. Disponível em:

http://repositorio.cepal.org/bitstream/handle/11 362/6407/1/S028593_es.pdf Acesso em: $13 / 10 / 2017$

FEIL, A. A.; STRASBURG, V. J.; SPILKI, F. R.; Variáveis intervenientes na existência de comitês de bacias hidrográficas no Brasil. 
Revista Ambiente \& Água, v. 12 n. 2, p. 340351, 2017. http://dx.doi.org/10.4136/ambiagua.1828

GLOBAL WATER PARTNERSHIP (GWP) Technical Advisory Committee. Integrated water resources management. TAC Background Paper, $\mathrm{n}^{\circ}$ 4, Stockholm, Sweden, 71p., 2000. Disponível em: https://www.gwp.org/globalassets/global/toolbo x/publications/background-papers/04-

integrated-water-resources-management-2000english.pdf Acesso 12/09/2017

HEINKE, J.; GERDEN, D.; HADDELAND, I.; ARNELL, N. W.; CLARK, D. B.; DANKERS, R.; EISNER, S.; FEKETE, B. M.; CÓLONGONAZÁLEZ, F. J.; GOSLING, S. N.; MASAKI, Y.; PORTMANN, F. T.; SATOH, Y.; TANG, Q.; WADA, Y.; WISSER, D.; FRIELER, K.; WARSZAWSKI, L.; KABAT, P.; Multimodel assessment of water scarcity under climate change. Proceedings of the National Academy of Sciences, PNAS, v. 111, n. 9, p.3245-3250, 2014.

http://dx.doi: org/10.1073/pnas. 1222460110

KAISER, I. M.; PORTO, R. de M.; Campos de Precipitação Parte I: Fundamentos Teóricos e Estudos Preliminares. Revista Brasileira de Recursos Hídricos, v. 10, n.4, p. 99-111, 2005. http://dx.doi: 10.21168/rbrh.v10n4.p99111

KORFMACHER, K. S; The politics of participation in watershed modeling. Environmental Management. v. 27, n. 2, p. 161-176, $2001 . \quad$ http://dx.doi: $10.1007 / \mathrm{s} 002670010141$

LANNA, A. E. L. Introdução à gestão ambiental e à análise econômica do ambiente. Porto Alegre: Instituto de Pesquisas Hidráulicas da UFRGS, 1996.

LANNA, A. E. L.; Modelos de gerenciamento das águas, a água em revista. Porto Alegre: CPRM, 1997.

LEIDEL, M.; NIEMANN, S.; HAGEMANN, N.; Capacity development as a key factor for integrated water resources management (IWRM): improving water management in the Western Bug River Basin, Ukraine. Environmental Earth Science, v. 65, p. 1415-1426, 2012. http://dx.doi:10.1007/s12665011-1223-5

NASCIMENTO, C. A.; STAGGEMEIER, R.; BIANCHI, E.; RODRIGUES, M. T.; FABRES, R.; SOLIMAN, M. C.; BORTOLUZZI, M. LUZ, R. B.; HEIZELMANN, L. S.; SANTOS, E. L.; FLECK, J. D.; SPILKI, F. R.; Monitoring of metals, organics compounds and coliforms in water catchment points from the Rivers Sinos basin. . Brazilian Journal Biological, v. 75, n.2, p. 50-56, 2015 . http://dx.doi.org/10.1590/1519-6984.1613

PETRY, C. T.; COSTA, G. M. da; BENVENUTI, T.; RODRIGUES, M. A. S.; DROSTE, A.; Avaliação integrada da qualidade química e da genotoxicidade da água do arroyo Luiz Rau, no trecho inferior da bacia do Rio dos Sinos, no Sul do Brasil. Ambiente \& Água, v.11, n.4, p 867-877, 2016. http://dx.doi:10.4136/ambiagua. 1779

PORTO, M. F. A.; PORTO, R. L L.; Gestão de bacias hidrográficas, Estudos Avançados, n. $22, \quad$ v. $63, \quad$ p. 43 - 60, 2008. http://dx.doi.org/10.1590/S010340142008000200004

SANTOS, L. C. D.; NHAMPOSSA, J. A.; COSTA, C. C.; GOMES, L. J.; Atuação do Comitê da Bacia Hidrográfica do Rio Sergipe na denúncia e encaminhamento de conflitos socioambientais. REGA, v. 12 , n. 2 , p. $35-45$, 2015. http://dx.doi: 10.21168/rega.v12n2.p35-45 SCHEWE, J.; HEINKE, J.; GERDEN, D.; HADDELAND, I.; ARNELL, N. W.; CLARK, D. B.; DANKERS, R.; EISNER, S.; FEKETE, B. M.; CÓLON-GONAZÁLEZ, F. J.; GOSLING, S. N.; MASAKI, Y.; PORTMANN, F. T.; SATOH, Y.; TANG, Q.; WADA, Y.; WISSER, D.; FRIELER, K.; WARSZAWSKI, L.; KABAT, P.; Multimodel assessment of water scarcity under climate change. Proceedings of the National Academy of Sciences, PNAS, v. 111, n. 9, p.3245-3250, 2014. http://dx.doi: org/10.1073/pnas. 1222460110

SENRA, J. B.; NASCIMENTO, N. O.; Após 20 anos da lei das águas como anda a Gestão Integrada de Recursos Hídricos do Brasil, no âmbito das Políticas e Planos Nacionais setoriais? REGA, v. 14, n. 6,18 p. 2017. http://dx.doi: 10.21168/rega.v.14e6

SHRUBSOLE, D.; WALTERS, D.; VEALE, B.; MITCHELL, B.; Integrated Water Resources Management in Canada: the experience of watershed agencies. International Journal of Water Resources Development. v. 33, p. 349-359, 2017. http://dx.doi: org/10.1080/07900627.2016.1244048

SICHANGI, A. W.; WANG, L.; YANG, K.; CHEN, D.; WANG, Z.; LI, X.; ZHOU, J.; LIU, W.; KURIA, D.; Estimating continental river basin discharges using multiple remote sensing data sets. Remote Sensing of Environment. v. 179, p. 36-53, 2016. http://dx.doi: org/10.1016/j.rse.2016.03.019

SISTEMA NACIONAL DE INFORMAÇÕES SOBRE RECURSOS HÍDRICOS (SNIRH) 
http://www.snirh.gov.br/hidroweb

STAHLI, M.; BADOUX, A.; LUDWIG, A.; STEINER, K.; ZAPPA, M.; HEGG, C.; One century of hydrological monitoring in two small catchments with different forest coverage. Environmental Monitoring Assessment, v. 174, p. 91-106. 2011. http://dx.doi: 10.1007/s10661-010-1757-0

RAUBER, D.; CRUZ, J. C.; Gestão de Recursos Hídricos: uma abordagem sobre os comitês de bacia hidrográfica. Revista Paranaense de Desenvolvimento, v. 34 , n. 125 , p. 123-140, $2013 . \quad$ Disponível em: http://www.ipardes.pr.gov.br/ojs/index.php/revi staparanaense/article/download/640/867

REIS, J. B. C. dos; PONS, N. A. D.; LOPES, E. S. S.; Monitoramento e alerta de inundação no município de Itajubá (MG) por regressão polinomial. Geociências, v. 35, n. 1, p.134148, 2016. Disponível em:http://www.ppegeo.igc.usp.br/index.php/GE OSP/article/view/9002/8267

RIBEIRO, C. B.; A importância dos comitês de bacia na gestão dos recursos hídricos. Dissertação de Mestrado em Geografia, área de concentração Gestão Ambiental e Territorial. Brasília - DF, 114 p., 2006. Disponível em: http://repositorio.unb.br/handle/10482/5754 Acesso em 10/09/2017

VEALE, B.; COOKE, S.; Implementing integrated water management: illustrations from the Grand River watershed. International Journal of Water Resources Development, v. 33, p. 375-392, 2017. http://dx.doi: org/10.1080/07900627.2016.1217503

WATANABE, M. M.; MADRUGA, L. R. G.; YAMAGUCHI, C. K.; VIEIRA, A. C. P.; JENOVEVA-NETO, R. Decision Making and Social Learning: the Case of Watershed Committee of the State of Rio Grande do Sul, Brazil. Water resources management, v. 28, n. $11, \quad$ p. 3815-3828, 2014 . http://dx.doi: org/10.1007/s11269-014-0711-2

WMO- WORLD METEREOLOGICAL ORGANIZATION; Guide to hydrological Practices, v.1, Hydrology - From Measurement to Hydrological Information, 296 p., 2008. Disponível em: http://www.whycos.org/chy/guide/168_Vol_I_en .pdf Acesso em: 01/05/2018

\footnotetext{
Guide to Hydrological Practices, v. 2, Sixth edition, Management of Water Resources and Application of Hydrological Practices. 302 p., 2009. Disponível em: http://www.whycos.org/chy/guide/168_Vol_II_e
}

n.pdf Acesso em: 01/05/2018

Manual on Stream Gauging. Fieldwork, v.1. Quality Management Framework. n. 1044. 254 p., 2010. Disponível em:

http://www.wmo.int/pages/prog/hwrp/publicati ons/stream_gauging/1044_Vol_I_en.pdf Acesso em: 01/05/2018

Planning of Water-Quality Monitoring Systems. Techinical Report Series, n. 3, 128 p., 2013. Disponível em: http://www.wmo.int/pages/prog/hwrp/publicati ons/Technical_report_series/TR-

No3water_quality_monitoring_systems.pdf Acesso em: 01/05/2018

- Guidelines for Hydrological Data Rescue, n 1146, 47 p., 2014. Disponível em: https://library.wmo.int/pmb_ged/wmo_1146_en .pdf Acesso em: 01/05/2018

$\begin{array}{ccc}\text { Guide to } & \text { Metereological } \\ \text { Instruments and methods of }\end{array}$ Observations. N.8, 1177p. 2017. Disponível em:

https://library.wmo.int/doc_num.php?explnum_ id $=4147$ Acesso em: 05/02/2020

WWAP - United Nations World Water Assessment Programme. The United Nations World Water Development Report 2017. Wastewater: The Untapped Resource. Paris, UNESCO, 198p., 2017. Disponível em: http://unesdoc.unesco.org/images/0024/002471/ 247153e.pdf Acesso em: 03/06/2018 